\title{
Expanding the palette
}

Adv. Electron. Mater. https://doi.org/10.1002/ aelm.201700600 (2018)

Fabricating electronic circuits on paper offers a sustainable route to wearable and healthcare technologies, and provides educators and artists with a means to teach and explore electronics. Depositing circuit components on paper typically requires the use of inkjet printing systems, and while research and commercial enterprises can fund such approaches, their cost is often a barrier to use in schools and by individuals. To alleviate the problem, inks with specific electrical properties have been developed that can be used in pens, enabling circuits to be drawn by hand. However, most demonstrations have so far been limited to drawing conductive electrodes and traces. Júlio Costa, Niko Münzenrieder and colleagues at the University of Sussex have now shown how a range of commercial conductive pens and graphite pencils can be used to fabricate hand drawn electronic components and circuits on standard printer paper.

The researchers used metal-based inks with different work functions to fabricate Schottky diodes, and graphite pencils to fabricate pressure-sensitive resistors and parallel plate capacitors that exploit the dielectric properties of the paper substrate. With the addition of a commercial operational amplifier, Münzenrieder and colleagues created a flexible paper-based pressure-sensing circuit that exhibits a sensitivity of $51 \mathrm{mV} \mathrm{kPa}{ }^{-1}$ and linear pressure sensing between 0.2 and $1.2 \mathrm{kPa}$. Furthermore, the approach can easily be used to draw circuits on three-dimensional structures, offering a low-cost and fun introduction to the world of electronics.

Stuart Thomas

Published online: 13 June 2018

https://doi.org/10.1038/s41928-018-0095-Z 